\title{
Relief Supplies Delivery Based on Path Connectivity Reliability
}

\author{
Hui $\mathrm{Hu}^{1}{ }^{1 *}$, Xing $\mathrm{Fu}^{1}$, Yang Cheng ${ }^{2}$ and Yunna Zhang ${ }^{1}$ \\ ${ }^{1}$ School of Automobile, Chang'an University, Xi'an 710064, China \\ ${ }^{2}$ Traffic Operations and Safety Lab, University of Wisconsin, Madison, Madison, WI 53706, United States
}

Received 22 August 2017; Accepted 30 November 2017

\begin{abstract}
To guarantee the safe and reliable delivery of relief supplies to disaster areas, a relief delivery model based on path connectivity reliability is proposed. In the study, the connectivity reliability of each path under various disruption levels and road grades was identified with Delphi method, and the possibility Goal Oriented (GO) method was introduced to identify the connectivity reliability of the delivery network. Based on the connectivity reliability of the network, a penalty parameter was introduced. Considering the uncertain influence of disruption on road network reliability, the interval number and triangular fuzzy number were used to indicate the uncertain delivery time and unit material delivery cost, respectively. A multi-objective model based on path connectivity reliability was established. Through the case study and result comparison, the study's improved model was proved to be superior. Results show that the delay times of the model and traditional model is 0 . Moreover, the delivery costs of the new model and traditional model are 17,586 and 17,340, respectively. Although the delivery cost of the new model is greater, its demand satisfaction rate is much higher than that of traditional models. Thus, the new model can significantly guide relief delivery when the road condition is affected by disruptions.
\end{abstract}

Keywords: Connectivity reliability, Relief delivery, Possibility GO method, Multi-objective optimization

\section{Introduction}

Disruptions have a serious impact on China's economic development and societal stability. In early 2008, 19 Chinese provinces suffered from snowstorms and persistent low temperature, which resulted in economic losses of 53.79 billion RMB [1]. The Wenchuan Earthquake in 2008 caused approximately 69,000 deaths and over 840 billion RMB in losses [2]. In 2015, the Tianjin explosion caused 165 deaths and 6.86 billion RMB worth of damages [3]. In other countries, the tsunami in Japan triggered by an earthquake resulted in 15,853 deaths and 300 billion dollars of losses in 2011. In Ecuador, the 7.8 magnitude earthquake killed more than 240 people and injured over 1,500 individuals in 2016 [4].

Relief supplies delivery is critical to the efficiency and effectiveness of rescue operations. Hence, scholars have gradually focused on relief delivery problems and have achieved some results [5-8]. The current research is based on known conditions of road networks. As an important index for measuring road network safety, the path connectivity reliability may be considered to immediately improve the satisfaction rate of relief supplies.

On the basis of the above analysis, the study proposes path connectivity reliability by using the Delphi and possibility Goal Oriented (GO) methods. Moreover, the study constructs an improved multi-objective model based on the path connectivity reliability with uncertain delivery times and unit material delivery costs. This model is valuable in improving the satisfaction rate of relief supplies and in

\footnotetext{
"E-mail address: huhui0414@163.com

ISSN: $1791-2377 \bigcirc 2017$ Eastern Macedonia and Thrace Institute of Technology. All rights reserved. doi:10.25103/iestr.106.07
}

guiding the reasonable planning of relief delivery affected by disruptions.

\section{State of the art}

Researchers have conducted extensive studies on relief delivery problems and constructed optimization models from operation research to minimize delivery times and costs and maximize satisfaction levels. Tzeng [5] constructed a fuzzy multi-objective relief delivery model to minimize cost and time and maximize satisfaction level, but the model did not consider road condition uncertainties. Sheu [6] presented a dynamic relief-demand management model for emergency logistics operations under imperfect information conditions in large-scale natural disasters, including data fusion, fuzzy clustering, and multi-criteria decision making, but the delivery model was not detailed. Given that relief delivery can be affected by damaged infrastructures, Nolz et al. [7] constructed a multi-objective integer optimization model to optimize safety, rescue coverage, and emergency time. Vitoriano et al. [8] studied humanitarian logistics through an objective programming approach from two aspects, specifically, the assessment of consequences in the early stage after a disaster and the end-phase distribution of humanitarian aid. Gan [9] constructed a relief delivery model on the basis of relief supplies urgency and introduced a divide-and-conquer algorithm to determine corresponding solutions. Berkoune [10] developed an efficient genetic algorithm to deal with realistic large-scale transportation problems in disasters. The algorithm provided high-quality transportation plans to emergency managers but did not consider relief delivery uncertainties. Zhao et al. [11] constructed an emergency material dispatching model that 
locates the earliest emergency time and least supply points. Zhang [12] constructed an optimization model to minimize emergency time and cost under material supply shortage context. Chen [13] established an emergency material dispatching optimization model within the emergency limitation period to obtain the shortest total delivery time under limited transportation capacity. The studies concentrated on relief delivery under certain conditions but did not discuss relief distribution uncertainties, such as road conditions, demand and supply, and delivery time, among others.

$\mathrm{Gu}$ [14] argued that optimization objective should be set as the minimum disaster loss parameter when relief supplies are urgently demanded. Emergency material dispatching models considered certain conditions and fuzzy situations, and delivery time uncertainties were resolved by selecting reliable paths in fuzzy networks. However, the study did not discuss the effect of disruptions on transportation conditions. Yang et al. [15] constructed a relief delivery model and described uncertain transportation time and demand by setting numerical intervals for the initial rescue stage, but the model did not relate uncertain transportation time with disruption conditions. In terms of material shortage in the initial stage of emergency material dispatch, Najafi et al. [16] proposed a stochastic model to manage emergency materials and developed a robust optimization method to ensure that the dispatching scheme was applicable to all earthquake situations. Wang et al. [17] constructed a nonlinear integer programming model by considering emergency time, emergency cost, and batch transportation reliability. The model adopted a non-dominant-sorted genetic algorithm and non-dominant sorting of differential evolution algorithm. Wang [18] studied the relief delivery problem under uncertain supply and demand conditions and constructed an optimization model that can determine minimum delivery time and maximum demand satisfaction levels. Yi [19] constructed an emergency material dispatching model based on the dynamic change of demand that can pinpoint the earliest emergency time at the minimum cost. Chen et al. [20] constructed a model by considering penalty and emergency cost parameters in the context of demand uncertainties. Although the studies identified uncertainties related to demand and supply, those uncertainties related to road transport conditions caused by disruptions were not considered. Previous studies also did not analyze the effect of disruption levels and road grades on road reliability.

Zhang et al. [21] constructed an emergency material dispatching model based on network reliability to minimize total shortage and emergency cost. The result of the modeling showed that multi-objective optimization was more economical and applicable than traditional models. He [22] evaluated a road network structure using improved genetic algorithm and designed an improved Dijkstra algorithm to find the best modeling solution.

In summary, existing research mainly focused on relief delivery problems associated with specific conditions or uncertain factors. Although some researchers analyzed the reliability of road networks, these scholars did not discuss the relationship among network reliability, disruption character, and road grade.

The remainder of this study is organized as follows: Section 3 introduces the detailed steps of determining the path connectivity reliability with the possibility GO method. Then, the multi-objective model based on path connectivity reliability is established. Section 4 discusses a case study and analyzes the results. Combined with actual data on the
Wenchuan earthquake, the delivery quantity, total delay time, and optimal total cost are determined. The proposed model is proven to be more superior than the traditional model. The last section on the summary of results concludes the study.

\section{Methodology}

The Delphi method is used to forecast path connectivity reliability with different disruption levels and road grades. Then, the road network is transferred to a GO graph, and the path connectivity reliability is obtained according to the possibility GO method calculation rule. From the calculations, the path with maximum reliability is established, and penalty coefficients are determined according to the connectivity reliability. The relief delivery model, the aim of which is to obtain minimum delay time and minimum cost, is constructed. This model can improve the satisfaction rate of relief supplies. Finally, the main objective method is introduced to convert the multi-objective model into a single-objective model with the shortest delay time. LINGO is used to find the optimal solution of the model.

\subsection{Problem Description}

$A_{1}, A_{2}, \cdots, A_{m}$ are supply points whereas $B_{1}, B_{2}, \cdots, B_{n}$ are disaster points. The road network is shown in Figure 1. $\mathrm{X}_{1}, \mathrm{X}_{2}, \cdots, \mathrm{X}_{\mathrm{w}}, \cdots, \mathrm{Y}_{1}, \mathrm{Y}_{2}, \cdots, \mathrm{Y}_{\mathrm{v}}$ are the nodes of the path between the supply and disaster points. The maximum supply point of $\mathrm{A}_{\mathrm{i}}(\mathrm{i}=1,2, \cdots, \mathrm{m})$ is $a_{i}$ whereas the demand of disaster points represented by $\mathrm{B}_{\mathrm{j}}(\mathrm{j}=1,2, \cdots, \mathrm{n})$ is $b_{j}$. Thus, $\sum_{i=1}^{m} a_{i} \geq \sum_{j=1}^{n} b_{j}$. The amount of relief supplies shipped from supply points to the disaster point is $x_{i j}$. Delivery time $\tilde{t}_{i j}$, a triangular fuzzy number, is expressed as $\tilde{t}_{i j}=\left[t_{i j 1}, t_{i j 2}, t_{i j 3}\right]$, where $t_{i j 1}$ is a pessimistic value of a fuzzy number, $t_{i j 2}$ is a normal value of a fuzzy number, and $t_{i j 3}$ is an optimistic value of a fuzzy number. The emergency deadline of disaster points $\mathrm{B}_{\mathrm{j}}$ is $t_{j}$, and thus, unit delay time is $\tilde{t}_{i j}-t_{j}$. Unit transportation cost $\tilde{c}_{i j}$, an interval number, is expressed as $\tilde{c}_{i j}=\left[c_{i j}^{-}, c_{i j}^{+}\right]$, where $c_{i j}^{-}$is the lower limit and $c_{i j}^{+}$is the upper limit. The maximum connectivity reliability $p_{i j}$ between $A_{i}$ and $B_{j}$ is obtained by using the possibility GO method. The penalty parameter corresponding to $p_{i j}$ is $r_{i j}$. From these expressions, the optimal relief delivery scheme is determined.

The model assumptions are as follows:

(1) Relief supplies in the supply points are adequate.

(2) Disaster areas need a certain kind of relief.

(3) All rescue activities occur at the period $t=1$.

(4) Connectivity reliability does not change during a unit period.

(5) Road capacity meets transportation requirements.

(6) Delay time is 0 when transportation time is less than emergency deadline. 


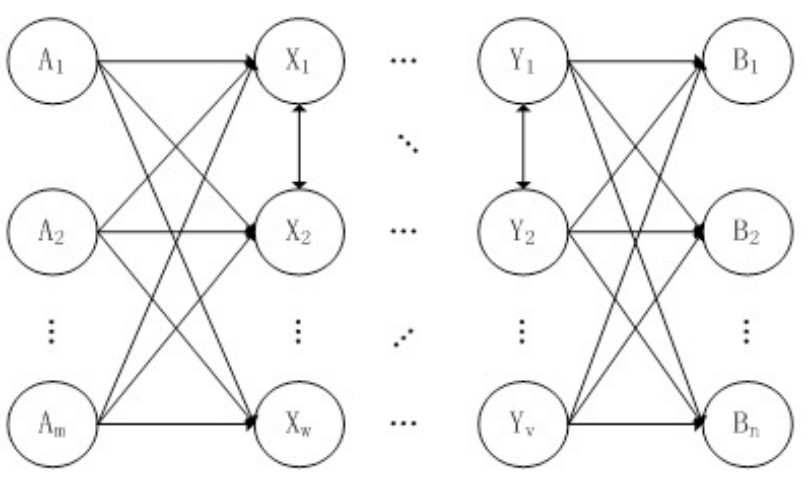

Fig. 1. Road network between supply points and disaster points

\subsection{Model formulation}

\subsubsection{Determination of path reliability}

Path connectivity reliability is usually evaluated from four aspects [23,24,25], namely, connectivity reliability, travel time reliability, capacity related reliability, and unblocking reliability. Relief supplies are not usually delivered to disaster points on time because road networks are not always connected. Thus, the state of road network is discussed in this study.

Path connectivity reliability is influenced by several factors, such as types of disruption, highway grade, and climatic conditions. Hence, the Delphi method is applied to predict the connectivity reliability of each route at different periods. Table 1 shows the result of path connectivity reliability with different disruption levels at period $t=1$.

Table 1. Path Connectivity Reliability with Different Disruption Levels

\begin{tabular}{c|c|c|c|c}
\hline Disruption Level & I & II & III & IV \\
\hline $\begin{array}{c}\text { Path Connectivity } \\
\text { Reliability } P_{t}\end{array}$ & 0.1815 & 0.4065 & 0.5380 & 0.7005 \\
\hline
\end{tabular}

Subsequently, the path connectivity reliability with different road levels is predicted.

Table 2. Path Connectivity Reliability with Different Road Grades

\begin{tabular}{c|c|c|c|c|c}
\hline Road Grade & Super & $\begin{array}{c}\text { First } \\
\text { Class }\end{array}$ & $\begin{array}{c}\text { Second } \\
\text { Class }\end{array}$ & $\begin{array}{c}\text { Third } \\
\text { Class }\end{array}$ & $\begin{array}{c}\text { Fourth } \\
\text { Class }\end{array}$ \\
\hline $\begin{array}{c}\text { Connectivity } \\
\text { Reliability } \\
P_{g}\end{array}$ & 0.7940 & 0.6690 & 0.5875 & 0.4005 & 0.1500 \\
\hline
\end{tabular}

After extensive investigation, path connectivity reliability is affected more by disruption level than road grade. Thus, the weight of $P_{t}$ is set to 0.7 whereas the weight of $P_{g}$ is set to 0.3 . Path connectivity reliability is expressed as:

$$
P=0.7 P_{t}+0.3 P_{g}
$$

The values of path connectivity reliability with different disruption levels and road grades is shown in Table 3.

Table 3. Path Connectivity Reliability by Disruption Level and Road Grade

\begin{tabular}{c|c|c|c|c}
\hline & \multicolumn{4}{|c}{ Disruption Level } \\
\hline Road Grade & I & II & III & IV \\
\hline Super & 0.61 & 0.68 & 0.72 & 0.77 \\
First Class & 0.52 & 0.59 & 0.63 & 0.68
\end{tabular}

\begin{tabular}{l|l|l|l|l} 
Second Class & 0.47 & 0.53 & 0.57 & 0.62 \\
Third Class & 0.33 & 0.40 & 0.44 & 0.49 \\
Forth Class & 0.16 & 0.23 & 0.27 & 0.32 \\
\hline
\end{tabular}

3.2.2 Path connectivity reliability determined by possibility GO method

The actual relief delivery network is transformed into a possibility GO diagram. On the basis of connectivity reliability of routes, the connectivity reliability of all paths is obtained according to the possibility GO method calculation rule. The path with the maximum connectivity reliability is then obtained.

\subsubsection{Penalty parameter determination}

The penalty parameters of relief supplies on various paths are given by experts on the basis of maximum connectivity reliability, relief supplies reserves, and vehicle quantity. The penalty parameters corresponding to different path connectivity reliabilities are shown in Table 4.

Table 4. Penalty Parameters for Different Path Connectivity Reliabilities

\begin{tabular}{c|c|c|c|c}
\hline $\begin{array}{c}\text { Connectivity } \\
\text { Reliability } \\
p_{i j}\end{array}$ & $0<p \leq 0.25$ & $0.25<p \leq 0.5$ & $0.5<p \leq 0.75$ & $0.75<p \leq 1$ \\
\hline $\begin{array}{c}\text { Penalty } \\
\text { Parameter } \\
r_{i j}\end{array}$ & 1 & 0.90 & 0.80 & 0.70 \\
\hline
\end{tabular}

\subsubsection{Model construction}

To minimize delay time and cost and to maximize satisfaction level of disaster points, the model is established by the following:

$\min T(x)=\min \sum_{j=1}^{n} \sum_{i=1}^{m} x_{i j} \cdot\left(\tilde{t}_{i j}-t_{j}\right)$

$\min C(x)=\min \sum_{j=1}^{n} \sum_{i=1}^{m} \tilde{c}_{i j} \cdot x_{i j}$

s.t. $\sum_{j=1}^{n} x_{i j} \leq a_{i}, \quad i=1,2, \mathrm{~L}, m$

$\sum_{i=1}^{m} r_{i j} \cdot x_{i j} \geq b_{j}, j=1,2, \mathrm{~L}, n$

$\tilde{t}_{i j}=\left[t_{i j 1}, t_{i j 2}, t_{i j 3}\right]$

$\tilde{c}_{i j}=\left[c_{i j}^{-}, c_{i j}^{+}\right]$

$x_{i j} \geq 0, \quad i=1,2, \mathrm{~L}, m ; j=1,2, \mathrm{~L}, n$

Equations (2) and (3) are objective functions for minimum total delay time and minimum cost. Equations (4) to (8) are constraint functions. Equation (4) suggests that the total amount of relief supplied to disaster point $B_{j}$ cannot exceed the maximum supply of supply point $A_{i}$, whereas Equation (5) suggests that the expected quantity of relief supplies for delivery should meet the requirements of 
disaster point $\mathrm{B}_{\mathrm{j}}$. In Equation (6), the triangular fuzzy number $\tilde{t}_{i j}$ is delivery time. In Equation (7), the interval number $\tilde{c}_{i j}$ is the unit material delivery cost. LINGO is used to find the optimal solution of the model.

\section{Result Analysis and Discussion}

\subsection{Case study}

An 8.0-magnitude earthquake occurred in Sichuan Province (China) on May 12, 2008, and the disaster resulted in huge losses. Wenchuan, Mianzhu, Beichuan, Qingchuan, Maoxian, Dujiangyan, Pingwu, Pengzhou, Jiangyou, and Deyang in the Sichuan Province were seriously damaged. Relief supplies from Chengdu, Mianyang, and Guangyuan needed to be delivered to the disaster areas.
The disaster points are represented by $\mathrm{B}_{1}, \mathrm{~B}_{2}, \mathrm{~B}_{3}, \mathrm{~B}_{4}, \mathrm{~B}_{5}, \mathrm{~B}_{6}, \mathrm{~B}_{7}, \mathrm{~B}_{8}, \mathrm{~B}_{9}, \mathrm{~B}_{10}, \mathrm{~B}_{11}$, and supply points are represented by $\mathrm{A}_{1}, \mathrm{~A}_{2}, \mathrm{~A}_{3}$. Transportation time $\tilde{t}_{i j}$ and unit transportation cost $\tilde{c}_{i j}$ are the original path attributes between supply points and disaster points (Table 5). The variable $\tilde{t}_{i j}$ (a triangular fuzzy number) is expressed as $\tilde{t}_{i j}=\left[t_{i j 1}, t_{i j 2}, t_{i j 3}\right]$ while $\tilde{c}_{i j}$ (interval numbers) is expressed by $\tilde{c}_{i j}=\left[c_{i j}^{-}, c_{i j}^{+}\right]$. The path grades of each path between disaster point $\mathrm{A}_{\mathrm{i}}$ and supply point $\mathrm{B}_{\mathrm{j}}$ are shown in Table 6 . Moreover, the expected delivery time to disaster point $B_{j}$ is $t_{1}=3, t_{2}=6, t_{3}=4, t_{4}=3, t_{5}=4, t_{6}=3, t_{7}=3, t_{8}=6, t_{9}=3$, $t_{10}=3$, and $t_{11}=4$. The maximum acceptable transportation cost is 20,000 and permissible error $\varepsilon$ is 0.01 .

Table 5. Original Path Attribute Values between $A_{i}$ and $B_{j}$

\begin{tabular}{|c|c|c|c|c|c|c|c|c|c|c|c|c|}
\hline & \multicolumn{2}{|c|}{$\mathrm{B}_{1}$} & \multicolumn{2}{|c|}{$\mathrm{B}_{2}$} & \multicolumn{2}{|c|}{$\mathrm{B}_{3}$} & \multicolumn{2}{|c|}{$\mathrm{B}_{4}$} & \multicolumn{2}{|c|}{$\mathrm{B}_{5}$} & \multicolumn{2}{|c|}{$\mathrm{B}_{6}$} \\
\hline & $t_{i 1}$ & $c_{i 1}$ & $t_{i 2}$ & $c_{i 2}$ & $t_{i 3}$ & $c_{i 3}$ & $t_{i 4}$ & $c_{i 4}$ & $t_{i 5}$ & $c_{i 5}$ & $t_{i 6}$ & $c_{i 6}$ \\
\hline$A_{1}$ & {$[3,4,5]$} & {$[2,4]$} & {$[1,2,3]$} & {$[1,3]$} & {$[2,3,4]$} & {$[3,5]$} & {$[6,7,8]$} & {$[6,8]$} & {$[1,2,3]$} & {$[1,3]$} & {$[1,2,3]$} & {$[1,3]$} \\
\hline $\mathrm{A}_{2}$ & {$[1,2,3]$} & {$[1,3]$} & {$[1,2,3]$} & {$[1,3]$} & {$[3,4,5]$} & {$[2,4]$} & {$[3,4,5]$} & {$[3,5]$} & {$[2,3,4]$} & {$[2,4]$} & {$[2,3,4]$} & {$[3,5]$} \\
\hline $\mathrm{A}_{3}$ & {$[6,7,8]$} & {$[6,8]$} & {$[6,7,8]$} & {$[6,8]$} & {$[4,5,6]$} & {$[5,7]$} & {$[2,3,4]$} & {$[1,3]$} & {$[6,7,8]$} & {$[6,8]$} & {$[7,8,9]$} & {$[7,9]$} \\
\hline Demand $b_{j}$ & \multicolumn{2}{|c|}{1,153} & \multicolumn{2}{|c|}{1,216} & \multicolumn{2}{|c|}{323} & \multicolumn{2}{|c|}{515} & \multicolumn{2}{|c|}{273} & \multicolumn{2}{|c|}{146} \\
\hline
\end{tabular}

\begin{tabular}{c|c|c|c|c|c|c|c|c|c|c}
\hline \multicolumn{2}{c|}{$\mathrm{B}_{7}$} & \multicolumn{2}{c|}{$\mathrm{B}_{8}$} & \multicolumn{2}{c|}{$\mathrm{B}_{9}$} & \multicolumn{2}{c|}{$\mathrm{B}_{10}$} & \multicolumn{2}{|c|}{$\mathrm{B}_{11}$} & Maximum Supply \\
\hline$t_{i 7}$ & $c_{i 7}$ & $t_{i 8}$ & $c_{i 8}$ & $t_{i 9}$ & $c_{i 9}$ & $t_{i 10}$ & $c_{i 10}$ & $t_{i 11}$ & $c_{i 11}$ & $a_{i}$ \\
\hline$[1,2,3]$ & {$[3,5]$} & {$[7,8,9]$} & {$[6,8]$} & {$[1,2,3]$} & {$[1,3]$} & {$[3,4,5]$} & {$[3,5]$} & {$[1,2,3]$} & {$[1,3]$} & 3,000 \\
{$[1,2,3]$} & {$[1,3]$} & {$[6,7,8]$} & {$[6,8]$} & {$[1,2,3]$} & {$[1,3]$} & {$[1,2,3]$} & {$[1,3]$} & {$[1,2,3]$} & {$[2,4]$} & 3,000 \\
{$[4,5,6]$} & {$[7,9]$} & {$[5,6,7]$} & {$[4,6]$} & {$[7,8,9]$} & {$[7,9]$} & {$[4,5,6]$} & {$[4,6]$} & {$[6,7,8]$} & {$[6,8]$} & 2,000 \\
\hline \multicolumn{2}{c|}{1,072} & \multicolumn{2}{|c|}{192} & \multicolumn{2}{|c|}{334} & 1,066 & \\
\hline
\end{tabular}

Table 6. Path Grade

\begin{tabular}{|c|c|c|c|c|c|c|c|}
\hline Path & Path Grade & Path & Path Grade & Path & Path Grade & Path & Path Grade \\
\hline $\mathrm{A}_{1}-\mathrm{B}_{6}$ & $\begin{array}{l}\text { Second Class } \\
\text { Highway }\end{array}$ & $\mathrm{B}_{2}-\mathrm{B}_{11}$ & $\begin{array}{c}\text { Third Class } \\
\text { Highway }\end{array}$ & $\mathrm{B}_{2}-\mathrm{B}_{7}$ & $\begin{array}{c}\text { Second Class } \\
\text { Highway }\end{array}$ & $\mathrm{A}_{2}-\mathrm{B}_{7}$ & $\begin{array}{c}\text { Super } \\
\text { ighway }\end{array}$ \\
\hline $\mathrm{A}_{1}-\mathrm{B}_{9}$ & $\begin{array}{l}\text { Second Class } \\
\text { Highway }\end{array}$ & $\mathrm{B}_{2}-\mathrm{B}_{3}$ & $\begin{array}{l}\text { Third Class } \\
\text { Highway }\end{array}$ & $\mathrm{B}_{3}-\mathrm{B}_{7}$ & $\begin{array}{l}\text { Third Class } \\
\text { Highway }\end{array}$ & $\mathrm{A}_{2}-\mathrm{B}_{10}$ & $\begin{array}{c}\text { Third Class } \\
\text { Highway }\end{array}$ \\
\hline $\mathrm{A}_{1}-\mathrm{B}_{11}$ & Super Highway & $\mathrm{B}_{9}-\mathrm{B}_{11}$ & $\begin{array}{c}\text { Second Class } \\
\text { Highway }\end{array}$ & $\mathrm{B}_{3}-\mathrm{B}_{8}$ & $\begin{array}{c}\text { Second Class } \\
\text { Highway }\end{array}$ & $\mathrm{A}_{2}-\mathrm{B}_{4}$ & $\begin{array}{c}\text { Third Class } \\
\text { Highway }\end{array}$ \\
\hline $\mathrm{B}_{1}-\mathrm{B}_{6}$ & $\begin{array}{l}\text { Second Class } \\
\text { Highway }\end{array}$ & $\mathrm{B}_{3}-\mathrm{B}_{10}$ & $\begin{array}{c}\text { Second Class } \\
\text { Highway }\end{array}$ & $\mathrm{B}_{7}-\mathrm{B}_{10}$ & Super Highway & $\mathrm{A}_{3}-\mathrm{B}_{4}$ & $\begin{array}{l}\text { Super } \\
\text { Highway }\end{array}$ \\
\hline $\mathrm{B}_{5}-\mathrm{B}_{6}$ & $\begin{array}{c}\text { Super } \\
\text { Highway }\end{array}$ & $\mathrm{B}_{4}-\mathrm{B}_{8}$ & $\begin{array}{l}\text { Third Class } \\
\text { Highway }\end{array}$ & $\mathrm{B}_{4}-\mathrm{B}_{10}$ & $\begin{array}{l}\text { Third Class } \\
\text { Highway }\end{array}$ & $A_{3}-B_{10}$ & $\begin{array}{l}\text { Super } \\
\text { Highway }\end{array}$ \\
\hline $\mathrm{B}_{6}-\mathrm{B}_{9}$ & $\begin{array}{l}\text { Third Class } \\
\text { Highway }\end{array}$ & $\mathrm{A}_{3}-\mathrm{B}_{2}$ & $\begin{array}{c}\text { Super } \\
\text { Highway }\end{array}$ & $\mathrm{A}_{2}-\mathrm{B}_{11}$ & $\begin{array}{c}\text { Super } \\
\text { Highway }\end{array}$ & $\mathrm{A}_{3}-\mathrm{B}_{8}$ & $\begin{array}{c}\text { Second Class } \\
\text { Highway }\end{array}$ \\
\hline $\mathrm{B}_{5}-\mathrm{B}_{6}$ & $\begin{array}{c}\text { Super } \\
\text { Highway }\end{array}$ & $\mathrm{A}_{3}-\mathrm{B}_{11}$ & $\begin{array}{c}\text { Super } \\
\text { Highway }\end{array}$ & $\mathrm{A}_{2}-\mathrm{B}_{2}$ & $\begin{array}{c}\text { Super } \\
\text { Highway }\end{array}$ & & \\
\hline
\end{tabular}

The delivery network between supply points $A_{1}, A_{2}, A_{3}$ and disaster points $\mathrm{B}_{1}, \mathrm{~B}_{2}, \mathrm{~B}_{3}, \mathrm{~B}_{4}, \mathrm{~B}_{5}, \mathrm{~B}_{6}, \mathrm{~B}_{7}, \mathrm{~B}_{8}, \mathrm{~B}_{9}, \mathrm{~B}_{10}, \mathrm{~B}_{11}$ is shown in Figure 2. 


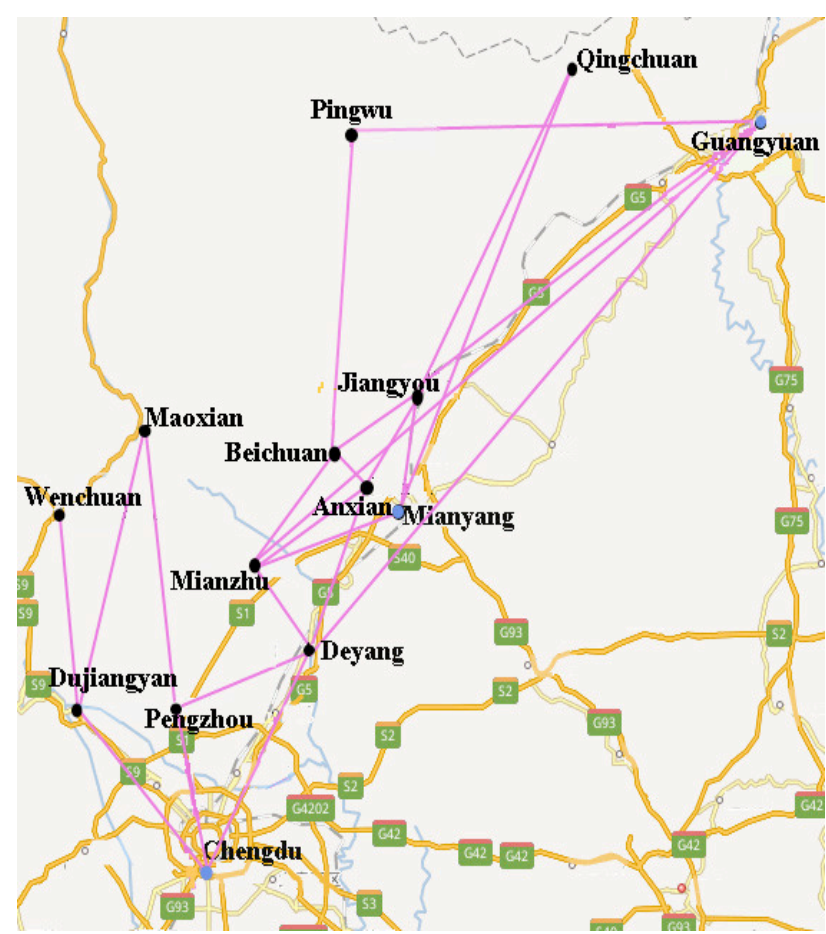

Fig. 2. Delivery Network

The connectivity reliability of each path, which can be obtained from Tables 3 and 6, is shown in Table 7.

Table 7. Path Connectivity Reliability of Each Pat

\begin{tabular}{c|c|c|c}
\hline Path & $\begin{array}{c}\text { Path } \\
\text { Connectivity } \\
\text { Reliability }\end{array}$ & Path & $\begin{array}{c}\text { Path } \\
\text { Connectivity } \\
\text { Reliability }\end{array}$ \\
\hline $\mathrm{A}_{1}-\mathrm{B}_{6}$ & 0.30 & $\mathrm{~B}_{2}-\mathrm{B}_{7}$ & 0.30 \\
$\mathrm{~A}_{1}-\mathrm{B}_{9}$ & 0.30 & $\mathrm{~B}_{3}-\mathrm{B}_{7}$ & 0.25 \\
$\mathrm{~A}_{1}-\mathrm{B}_{11}$ & 0.37 & $\mathrm{~B}_{3}-\mathrm{B}_{8}$ & 0.30 \\
$\mathrm{~B}_{1}-\mathrm{B}_{6}$ & 0.30 & $\mathrm{~B}_{7}-\mathrm{B}_{10}$ & 0.37 \\
$\mathrm{~B}_{5}-\mathrm{B}_{6}$ & 0.37 & $\mathrm{~B}_{4}-\mathrm{B}_{10}$ & 0.25 \\
$\mathrm{~B}_{6}-\mathrm{B}_{9}$ & 0.25 & $\mathrm{~A}_{2}-\mathrm{B}_{11}$ & 0.37 \\
$\mathrm{~B}_{5}-\mathrm{B}_{6}$ & 0.37 & $\mathrm{~A}_{2}-\mathrm{B}_{2}$ & 0.37 \\
$\mathrm{~B}_{2}-\mathrm{B}_{11}$ & 0.25 & $\mathrm{~A}_{2}-\mathrm{B}_{7}$ & 0.37 \\
$\mathrm{~B}_{2}-\mathrm{B}_{3}$ & 0.25 & $\mathrm{~A}_{2}-\mathrm{B}_{10}$ & 0.25 \\
$\mathrm{~B}_{9}-\mathrm{B}_{11}$ & 0.30 & $\mathrm{~A}_{2}-\mathrm{B}_{4}$ & 0.25 \\
$\mathrm{~B}_{3}-\mathrm{B}_{10}$ & 0.30 & $\mathrm{~A}_{3}-\mathrm{B}_{4}$ & 0.37 \\
$\mathrm{~B}_{4}-\mathrm{B}_{8}$ & 0.25 & $\mathrm{~A}_{3}-\mathrm{B}_{10}$ & 0.37 \\
$\mathrm{~A}_{3}-\mathrm{B}_{2}$ & 0.37 & $\mathrm{~A}_{3}-\mathrm{B}_{8}$ & 0.30 \\
$\mathrm{~A}_{3}-\mathrm{B}_{11}$ & 0.37 & & \\
\hline
\end{tabular}

Figures 3 to 5 are GO diagrams converted from Figure 2 . In the figures, the input nodes $\mathrm{A}_{1}, \mathrm{~A}_{2}, \mathrm{~A}_{3}$ are denoted by the type 5 operator; the "logical OR" relation of signals are denoted by the type 2 operator; each path is denoted by the type 1 operator; and each node is denoted by the type 13 operator. The dashed-line arrow in the figure suggests that signal flow can only move in one direction and cannot form a loop. The first number on the GO operator represents the operator type whereas the second number represents the operator number.

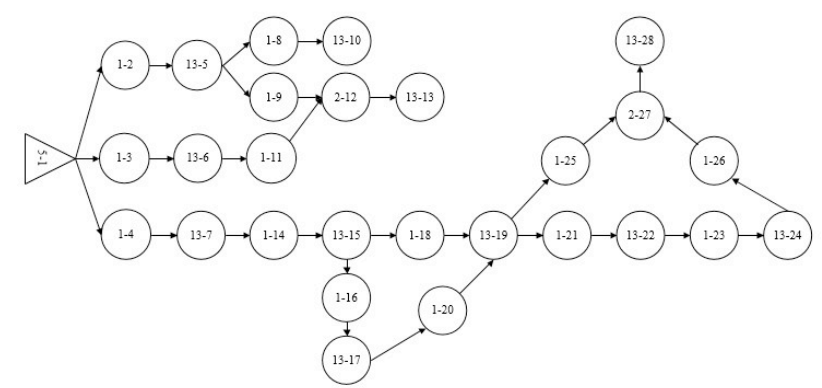

Fig. 3. GO diagram between Supply Point $A_{1}$ and different disaster points

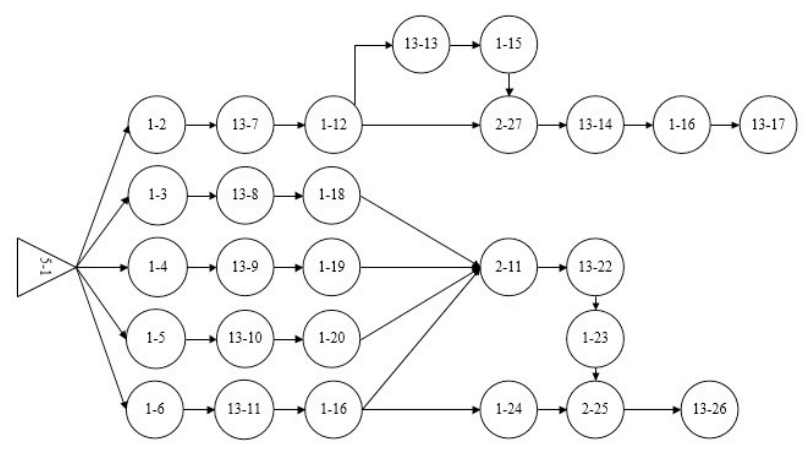

Fig. 4. GO diagram between Supply Point $A_{2}$ and different disaster points

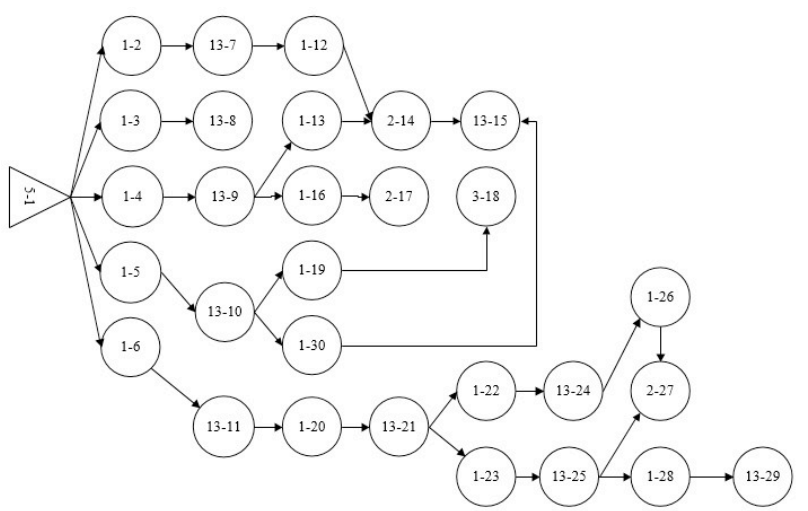

Fig. 5. GO diagram between Supply Point $A_{3}$ and different disaster points

The maximum path connectivity reliability in the network can be obtained by the possibility GO method calculation rule. Results are shown in Table 8 . The penalty parameters corresponding to maximum path connectivity reliability values are shown in Table 9.

During calculation, the triangular fuzzy number $\tilde{t}_{i j}$ is transformed into a definite real number. In addition, assuming the decision makers are a neutral type, the interval number $\tilde{c}_{i j}$ can be transformed into a deterministic real number. Accordingly, the path optimization attribute values can be obtained, and the numerical results are shown in Table 10.

Table 8. Maximum Path Connectivity Reliability in the Delivery Network

\begin{tabular}{c|c|c|c}
\hline Path & $\begin{array}{c}\text { Path } \\
\text { Connectivity } \\
\text { Reliability }\end{array}$ & Path & $\begin{array}{c}\text { Path } \\
\text { Connectivity } \\
\text { Reliability }\end{array}$ \\
\cline { 1 - 1 } $\mathrm{A}_{1}-\mathrm{B}_{1}$ & 0.30 & $\mathrm{~A}_{2}-\mathrm{B}_{7}$ & 0.37 \\
\hline
\end{tabular}




\begin{tabular}{|c|c|c|c|}
\hline$A_{1}-B_{2}$ & 0.25 & $\mathrm{~A}_{2}-\mathrm{B}_{8}$ & 0.25 \\
\hline$A_{1}-B_{3}$ & 0.25 & $\mathrm{~A}_{2}-\mathrm{B}_{9}$ & 0.30 \\
\hline$A_{1}-B_{4}$ & 0.25 & $\mathrm{~A}_{2}-\mathrm{B}_{10}$ & 0.25 \\
\hline $\mathrm{A}_{1}-\mathrm{B}_{5}$ & 0.30 & $\mathrm{~A}_{2}-\mathrm{B}_{11}$ & 0.37 \\
\hline $\mathrm{A}_{1}-\mathrm{B}_{6}$ & 0.30 & $\mathrm{~A}_{3}-\mathrm{B}_{1}$ & 0.30 \\
\hline$A_{1}-B_{7}$ & 0.25 & $\mathrm{~A}_{3}-\mathrm{B}_{2}$ & 0.37 \\
\hline$A_{1}-B_{8}$ & 0.25 & $\mathrm{~A}_{3}-\mathrm{B}_{3}$ & 0.25 \\
\hline $\mathrm{A}_{1}-\mathrm{B}_{9}$ & 0.30 & $\mathrm{~A}_{3}-\mathrm{B}_{4}$ & 0.37 \\
\hline$A_{1}-B_{10}$ & 0.25 & $\mathrm{~A}_{3}-\mathrm{B}_{5}$ & 0.30 \\
\hline$A_{1}-B_{11}$ & 0.37 & $\mathrm{~A}_{3}-\mathrm{B}_{6}$ & 0.30 \\
\hline$A_{2}-B_{1}$ & 0.25 & $\mathrm{~A}_{3}-\mathrm{B}_{7}$ & 0.30 \\
\hline $\mathrm{A}_{2}-\mathrm{B}_{2}$ & 0.37 & $\mathrm{~A}_{3}-\mathrm{B}_{8}$ & 0.25 \\
\hline $\mathrm{A}_{2}-\mathrm{B}_{3}$ & 0.25 & $\mathrm{~A}_{3}-\mathrm{B}_{9}$ & 0.30 \\
\hline $\mathrm{A}_{2}-\mathrm{B}_{4}$ & 0.25 & $\mathrm{~A}_{3}-\mathrm{B}_{10}$ & 0.37 \\
\hline $\mathrm{A}_{2}-\mathrm{B}_{5}$ & 0.30 & $\mathrm{~A}_{3}-\mathrm{B}_{11}$ & 0.37 \\
\hline $\mathrm{A}_{2}-\mathrm{B}_{6}$ & 0.30 & & \\
\hline
\end{tabular}

Table 9. Maximum Path Connectivity Reliability in the Delivery Network

\begin{tabular}{c|c|c|c}
\hline Path & $\begin{array}{c}\text { Penalty } \\
\text { Parameter }\end{array}$ & Path & $\begin{array}{c}\text { Penalty } \\
\text { Parameter }\end{array}$ \\
\hline $\mathrm{A}_{1}-\mathrm{B}_{1}$ & 0.9 & $\mathrm{~A}_{2}-\mathrm{B}_{7}$ & 0.9 \\
$\mathrm{~A}_{1}-\mathrm{B}_{2}$ & 1 & $\mathrm{~A}_{2}-\mathrm{B}_{8}$ & 1 \\
$\mathrm{~A}_{1}-\mathrm{B}_{3}$ & 1 & $\mathrm{~A}_{2}-\mathrm{B}_{9}$ & 0.9 \\
$\mathrm{~A}_{1}-\mathrm{B}_{4}$ & 1 & $\mathrm{~A}_{2}-\mathrm{B}_{10}$ & 1 \\
$\mathrm{~A}_{1}-\mathrm{B}_{5}$ & 0.9 & $\mathrm{~A}_{2}-\mathrm{B}_{11}$ & 0.9 \\
$\mathrm{~A}_{1}-\mathrm{B}_{6}$ & 0.9 & $\mathrm{~A}_{3}-\mathrm{B}_{1}$ & 0.9 \\
$\mathrm{~A}_{1}-\mathrm{B}_{7}$ & 1 & $\mathrm{~A}_{3}-\mathrm{B}_{2}$ & 0.9 \\
$\mathrm{~A}_{1}-\mathrm{B}_{8}$ & 1 & $\mathrm{~A}_{3}-\mathrm{B}_{3}$ & 1 \\
$\mathrm{~A}_{1}-\mathrm{B}_{9}$ & 0.9 & $\mathrm{~A}_{3}-\mathrm{B}_{4}$ & 0.9 \\
$\mathrm{~A}_{1}-\mathrm{B}_{10}$ & 1 & $\mathrm{~A}_{3}-\mathrm{B}_{5}$ & 0.9 \\
$\mathrm{~A}_{1}-\mathrm{B}_{11}$ & 1 & $\mathrm{~A}_{3}-\mathrm{B}_{6}$ & 0.9 \\
$\mathrm{~A}_{2}-\mathrm{B}_{1}$ & 1 & $\mathrm{~A}_{3}-\mathrm{B}_{7}$ & 0.9 \\
$\mathrm{~A}_{2}-\mathrm{B}_{2}$ & 0.9 & $\mathrm{~A}_{3}-\mathrm{B}_{8}$ & 1 \\
$\mathrm{~A}_{2}-\mathrm{B}_{3}$ & 1 & $\mathrm{~A}_{3}-\mathrm{B}_{9}$ & 0.9 \\
$\mathrm{~A}_{2}-\mathrm{B}_{4}$ & 1 & $\mathrm{~A}_{3}-\mathrm{B}_{10}$ & 0.9 \\
$\mathrm{~A}_{2}-\mathrm{B}_{5}$ & 0.9 & $\mathrm{~A}_{3}-\mathrm{B}_{11}$ & 0.9 \\
$\mathrm{~A}_{2}-\mathrm{B}_{6}$ & 0.9 & & \\
\hline & & & \\
\hline
\end{tabular}

Table 10. Path Optimization Attribute Values

\begin{tabular}{c|c|c|c|c|c|c|c|c}
\hline & \multicolumn{2}{|c|}{$\mathrm{B}_{1}$} & \multicolumn{2}{c|}{$\mathrm{B}_{2}$} & \multicolumn{2}{|c|}{$\mathrm{B}_{3}$} & \multicolumn{2}{|c}{$\mathrm{B}_{4}$} \\
\hline & $t_{i 1}$ & $c_{i 1}$ & $t_{i 2}$ & $c_{i 2}$ & $t_{i 3}$ & $c_{i 3}$ & $t_{i 4}$ & $c_{i 4}$ \\
\hline $\mathrm{A}_{1}$ & 1 & 3 & 0 & 2 & 1 & 4 & 1 & 7 \\
$\mathrm{~A}_{2}$ & 0 & 2 & 0 & 2 & 0 & 3 & 1 & 4 \\
$\mathrm{~A}_{3}$ & 1 & 7 & 1 & 7 & 1 & 6 & 0 & 2 \\
\hline Demand & \multicolumn{2}{|c|}{1,153} & \multicolumn{2}{|c|}{1,216} & \multicolumn{2}{|c|}{323} & \multicolumn{2}{|c}{515} \\
$b_{j}$ & \multicolumn{2}{|c}{} \\
\hline
\end{tabular}

\begin{tabular}{c|c|c|c|c|c|c|c}
\hline \multicolumn{2}{c|}{$\mathrm{B}_{5}$} & \multicolumn{2}{c|}{$\mathrm{B}_{6}$} & \multicolumn{2}{c|}{$\mathrm{B}_{7}$} & \multicolumn{2}{c}{$\mathrm{B}_{8}$} \\
\hline$t_{i 5}$ & $c_{i 5}$ & $t_{i 6}$ & $c_{i 6}$ & $t_{i 7}$ & $c_{i 7}$ & $t_{i 8}$ & $c_{i 8}$ \\
0 & 2 & 0 & 2 & 1 & 4 & 1 & 7 \\
0 & 3 & 1 & 4 & 0 & 2 & 1 & 7 \\
1 & 7 & 1 & 8 & 1 & 8 & 0 & 5 \\
\hline
\end{tabular}

\begin{tabular}{|c|c|c|c|c|c|c|}
\hline \multicolumn{2}{|c|}{273} & \multicolumn{2}{|c|}{146} & \multicolumn{2}{|c|}{449} & 1,072 \\
\hline \multicolumn{2}{|c|}{$\mathrm{B}_{9}$} & \multicolumn{2}{|c|}{$\mathrm{B}_{10}$} & \multicolumn{2}{|c|}{$\mathrm{B}_{11}$} & $\begin{array}{l}\text { Maximum } \\
\text { Supply }\end{array}$ \\
\hline$t_{i 9}$ & $c_{i 9}$ & $t_{i 10}$ & $c_{i 10}$ & $t_{i 11}$ & $c_{i 11}$ & $a_{i}$ \\
\hline 0 & 2 & 1 & 4 & 0 & 2 & 3,000 \\
\hline 0 & 2 & 0 & 2 & 0 & 3 & 3,000 \\
\hline 1 & 8 & 0 & 5 & 1 & 7 & 2,000 \\
\hline \multicolumn{2}{|c|}{$\frac{1}{192}$} & \multicolumn{2}{|c|}{$\frac{1}{334}$} & \multicolumn{2}{|c|}{$\frac{1}{1,066}$} & \\
\hline
\end{tabular}

Based on the above parameters, the optimization model of the case is as follows:

$$
\begin{aligned}
& \min T(x)=x_{1}+x_{3}+x_{4}+x_{7}+x_{8}+x_{10}+ \\
& \mathrm{y}_{4}+y_{6}+y_{8}+z_{1}+z_{2}+z_{3}+z_{5} \\
& +z_{6}+z_{7}+z_{9}+z_{10}+z_{11} \\
& \min C(x)=3 x_{1}+2 x_{2}+3 x_{3}+7 x_{4}+2 x_{5} \\
& +2 x_{6}+2 x_{7}+6 x_{8}+2 x_{9}+4 x_{10} \\
& +2 x_{11}+2 \mathrm{y}_{1}+2 \mathrm{y}_{2}+4 \mathrm{y}_{3}+4 \mathrm{y}_{4}+ \\
& 3 \mathrm{y}_{5}+3 \mathrm{y}_{6}+2 \mathrm{y}_{7}+7 y_{8}+2 \mathrm{y}_{9}+ \\
& 2 \mathrm{y}_{10}+2 \mathrm{y}_{11}+7 z_{1}+7 z_{2}+6 z_{3}+ \\
& 2 z_{4}+7 z_{5}+8 z_{6}+8 z_{7}+5 z_{8}+8 z_{9} \\
& +5 z_{10}+7 z_{11} \\
& 0.9 x_{1}+\mathrm{y}_{1}+0.9 z_{1} \geq 1153 \\
& 0.9 x_{2}+y_{2}+0.9 z_{2} \geq 1216 \\
& x_{3}+\mathrm{y}_{3}+z_{3} \geq 323 \\
& x_{4}+\mathrm{y}_{4}+0.9 z_{4} \geq 515 \\
& 0.9 x_{5}+0.9 y_{5}+0.9 z_{5} \geq 273 \\
& 0.9 x_{6}+0.9 \mathrm{y}_{6}+0.9 z_{6} \geq 146 \\
& \text { s.t. }\left\{\begin{array}{l}
x_{7}+0.9 y_{7}+0.9 z_{7} \geq 449 \\
x_{8}+y_{8}+z_{8} \geq 1072
\end{array}\right. \\
& 0.9 x_{9}+0.9 y_{9}+0.9 z_{9} \geq 192 \\
& x_{10}+\mathrm{y}_{10}+0.9 z_{10} \geq 334 \\
& 0.9 x_{11}+0.9 \mathrm{y}_{11}+0.9 z_{11} \geq 1066 \\
& x_{1}+x_{2}+x_{3}+x_{4}+x_{5}+x_{6}+x_{7}+x_{8}+x_{9}+x_{10}+x_{11} \leq 3000 \\
& \mathrm{y}_{1}+\mathrm{y}_{2}+\mathrm{y}_{3}+\mathrm{y}_{4}+\mathrm{y}_{5}+y_{6}+\mathrm{y}_{7}+y_{8}+\mathrm{y}_{9}+\mathrm{y}_{10}+\mathrm{y}_{11} \leq 3000 \\
& z_{1}+z_{2}+z_{3}+z_{4}+z_{5}+z_{6}+z_{7}+z_{8}+z_{9}+z_{10}+z_{11} \leq 2000
\end{aligned}
$$

To facilitate calculation, the main objective method is introduced when transforming the multiple objective model into a single-objective model. The single-objective model is solved by LINGO.

$x=\left[\begin{array}{ccccccccccc}0 & 1216 & 0 & 0 & 222 & 163 & 0 & 0 & 214 & 0 & 1185 \\ 1153 & 0 & 323 & 0 & 82 & 0 & 499 & 0 & 0 & 334 & 0 \\ 0 & 0 & 0 & 573 & 0 & 0 & 0 & 1072 & 0 & 0 & 0\end{array}\right]$

The optimal objective value is:

$T(x)=0, \quad C(x)=17586$

\subsection{Comparative Analysis}

\subsubsection{Comparison with traditional model}

The traditional model does not consider connectivity reliability. With the same parameters, the results of 
modeling proposed in this study are shown in Equations (14) and (15).

$$
\begin{aligned}
& \min T(x)=x_{1}+x_{3}+x_{4}+x_{7}+x_{8}+x_{10}+ \\
& \mathrm{y}_{4}+y_{6}+y_{8}+z_{1}+z_{2}+z_{3}+z_{5} \\
& +z_{6}+z_{7}+z_{9}+z_{10}+z_{11} \\
& \min C(x)=3 x_{1}+2 x_{2}+3 x_{3}+7 x_{4}+2 x_{5}+ \\
& 2 x_{6}+2 x_{7}+6 x_{8}+2 x_{9}+4 x_{10}+ \\
& 2 \mathrm{x}_{11}+2 \mathrm{y}_{1}+2 \mathrm{y}_{2}+4 \mathrm{y}_{3}+4 \mathrm{y}_{4}+ \\
& 3 \mathrm{y}_{5}+3 y_{6}+2 \mathrm{y}_{7}+7 y_{8}+2 \mathrm{y}_{9}+ \\
& 2 \mathrm{y}_{10}+2 \mathrm{y}_{11}+7 z_{1}+7 z_{2}+6 z_{3}+ \\
& 2 \mathrm{z}_{4}+7 z_{5}+8 z_{6}+8 z_{7}+5 z_{8}+ \\
& 8 z_{9}+5 z_{10}+7 z_{11} \\
& \text { s.t. }\left\{\begin{array}{l}
x_{1}+y_{1}+z_{1} \geq 1153 \\
x_{2}+y_{2}+z_{2} \geq 1216 \\
x_{3}+y_{3}+z_{3} \geq 323 \\
x_{4}+y_{4}+z_{4} \geq 515 \\
x_{5}+y_{5}+z_{5} \geq 273 \\
x_{6}+y_{6}+z_{6} \geq 146 \\
x_{7}+y_{7}+z_{7} \geq 449 \\
x_{8}+y_{8}+z_{8} \geq 1072 \\
x_{9}+y_{9}+z_{9} \geq 192 \\
x_{10}+y_{10}+z_{10} \geq 334 \\
x_{11}+y_{11}+z_{11} \geq 1066 \\
x_{1}+x_{2}+x_{3}+x_{4}+x_{5}+x_{6}+x_{7}+x_{8}+x_{9}+x_{10}+x_{11} \leq 3000 \\
y_{1}+y_{2}+y_{3}+y_{4}+y_{5}+y_{6}+y_{7}+y_{8}+y_{9}+y_{10}+y_{11} \leq 3000 \\
z_{1}+z_{2}+z_{3}+z_{4}+z_{5}+z_{6}+z_{7}+z_{8}+z_{9}+z_{10}+z_{11} \leq 2000
\end{array}\right.
\end{aligned}
$$

Similarly, LINGO is used to find the optimal solution.

$$
x=\left[\begin{array}{ccccccccccc}
0 & 1216 & 0 & 0 & 273 & 146 & 0 & 0 & 192 & 0 & 1066 \\
1153 & 0 & 323 & 0 & 0 & 0 & 449 & 0 & 0 & 334 & 0 \\
0 & 0 & 0 & 515 & 0 & 0 & 0 & 1072 & 0 & 0 & 0
\end{array}\right]
$$

The optimal objective value is:

$$
T(x)=0, \quad C(x)=17340
$$

A comparison of Equations (13) and (18) shows that the delay times are both 0 and the transportation costs are 17,586 and 17,340. In other words, all relief supplies can be delivered to disaster points at an emergency-limit period. Moreover, transportation costs are within acceptable range. However, when the path between supply point $A_{1}$ and disaster point $B_{5}$ is interrupted, the demand satisfaction levels of disaster points of the two models differ. The demand satisfaction levels of the traditional model and the proposed model are expressed by $R_{B_{j}}$ and $R_{B_{j}}^{\prime}$, respectively. The functions are expressed as $R_{B_{5}}=\frac{0}{273}=0$ and $\mathrm{R}_{\mathrm{B}_{5}}^{\prime}=\frac{82}{273}=0.3$. The proposed model obtained a higher satisfaction rate than the traditional model. Moreover, the proposed model can offer additional advantages in terms of generated supply points, disaster points, and paths. The delivery of relief supplies on the basis of path connectivity reliability not only improves the proper identification of disaster points, but also avoids substantial increase in transport costs.

\subsubsection{Comparison with other results from literature}

The proposed model is compared with the results from literature (26), and the differences are shown in Table 11.

Several studies on minimum cost and minimum delay time have been conducted. However, the demand satisfaction rates of disaster areas are often ignored. By contrast, the proposed model in this study not only considers minimum cost and delay time, but also introduces a penalty parameter. With the penalty parameter, an appropriate number of relief supplies is delivered, and accordingly, the demand satisfaction rate of the disaster points is increased. As shown in Table 11, the transportation cost and maximum delivery time in this study are superior to the results from literature [26].

Table 11. Comparison with other results

\begin{tabular}{|c|c|c|c|c}
\hline & $\begin{array}{c}\text { Proposed } \\
\text { model } \\
\text { (this study) }\end{array}$ & $\begin{array}{c}\text { Scenari } \\
\mathbf{0 1}\end{array}$ & $\begin{array}{c}\text { Scenari } \\
\mathbf{0 ~ 2}\end{array}$ & $\begin{array}{c}\text { Scenari } \\
\mathbf{0 3}\end{array}$ \\
\hline $\begin{array}{c}\text { Transportation } \\
\begin{array}{c}\text { Cost } \\
\text { Maximum } \\
\text { Delivery Time }\end{array}\end{array}$ & 17,686 & 18,683 & 18,242 & 9.3 \\
\hline
\end{tabular}

\section{Conclusions}

To improve the satisfaction rate of relief supplies in disaster areas, and to establish the impact of disruption levels and road grades on relief delivery, the path connectivity reliability was introduced in the present study. Path connectivity reliability was identified by using the Delphi method and the possibility GO method, and a multiobjective model was established after introducing the penalty parameter. Consequently, the proposed model minimized delay time and cost. The following conclusions could be drawn:

(1) Disruption level and road grade affect relief delivery in varying degrees. A relatively higher disruption level and a lower road grade can cause great impact to relief delivery.

(2) From the path connectivity reliability calculations, the path with relatively higher reliability may be chosen as an option for relief delivery, assuming the appropriate amount of relief supplies is allowed. Moreover, the relief satisfaction rate of the proposed model is higher than that of the traditional model.

(3) The transportation cost and maximum delivery time generated by this study are both superior compared with results from literature [26]. Findings imply that the proposed model can provide optimal solutions in terms of cost and time, and the model can generate high satisfaction rate of relief supplies.

The path connectivity reliability is capable of solving relief delivery problems due to uncertain transportation time and unit-level transportation costs. This study can significantly enhance the satisfaction rate of relief supplies and provide a reference for scientific relief delivery planning However, dynamic changes in road conditions are not considered in the proposed model. In future studies, GPS and GIS will be introduced to provide real-time information 
on road networks to help determine the dynamic optimal solution to relief delivery.

\section{Acknowledgements}

This work was supported by the National Natural Science Foundation of China (61503043) and Shaanxi Natural Science Foundation (2017JM7016).
This is an Open Access article distributed under the terms of the Creative Commons Attribution Licence

\section{References}

1. Zhao, W. W., Wu, G. Z., "Characteristics of snow disasters in south China and countermeasures for urban disaster relief". China Safety Science Journal, 18(10), 2008, pp.5-9.

2. Yan, G. M., "Reliable path search of emergency logistics under disruptions". Master thesis of Huazhong University of Science and Technology, China, 2010, pp.20-40.

3. Li, A. N., Deng, X. Q., Zhao, Q. H., "Unconventional emergency organizational dynamic reconstruction based on Fractal-8.12 Tianjin Explosions case study”. Management Review, 28(8), 2016, pp.193-206.

4. Xu, A. H., Chen, H., "Disaster of Ecuador earthquake and the emergency response and rescue". Recent Developments in World Seismology, 450(6), 2016, pp.26-31.

5. Tzeng, G. H., Cheng, H. J., Huang, T. D., "Multi-objective optimal planning for designing relief delivery systems". Transportation Research Part E: Logistics \& Transportation Review, 43(6), 2007, pp.673-686.

6. Sheu, J. B., "Dynamic relief-demand management for emergency logistics operations under large-scale disasters". Transportation Research Part E: Logistics \& Transportation Review, 46(1), 2010, pp.1-17.

7. Nolz, P. C., Semet, F., Doerner. K. F., "Risk approaches for delivering disaster relief supplies". OR Spectrum, 33(3), 2011, pp.543-569.

8. Vitoriano, B., Rodrguez, J. T., Tirado, G., "Intelligent decisionmaking models for disaster management". Human and Ecological Risk Assessment, 21(5), 2015, pp.1341-1360

9. Gan, Y., Lu, S. L., Li, J. X., "Research on emergency dispatching based on multi-depot, multi-material and cost". China Safety Science Journal, 21(9), 2011, pp.172-176.

10. Berkoune, D., Renaud, J., Rekik, M., "Transportation in disaster response operations". Socio-Economic Planning Sciences, 46(1), 2012, pp.23-32.

11. Zhao, M., Song, X. Y., Dong, J., "Using Genetic Algorithm to solve scheduling optimization problem of emergency supplies". Journal of Shenyang Jianzhu University Natural Science, 28(5), 2012, pp.944-948.

12. Zhang, S., "Relief supplies delivery under unconventional event". Master thesis of Yanshan University, China, 2013, pp.30-50.

13. Chen, J. J., "Scheduling model of multiple materials for marine casualties based on priority of scheduling". Journal of Zhejiang Ocean University (Natural Science), 37(4), 2014, pp.377-381.
14. Gu, Y., "Research on Optimization of Relief Supplies Distribution Aimed to Minimize Disaster Losses". Journal of Computers, 6(3), 2011, pp.603-609.

15. Yang, J. N., Yang, W. P., "Relief supplies delivery model of initial phase". China Market, 36(9), 2012, pp.9-11.

16. Najafi, M., Eshghi, K., Dullaert, W., "A multi-objective robust optimization model for logistics planning in the earthquake response phase". Transportation Research Part E: Logistics \& Transportation Review, 49(1), 2013, pp.217-249.

17. Wang, H., Du, L., Ma, S., "Multi-objective open location-routing model with split delivery for optimized relief distribution in postearthquake". Transportation Research Part E: Logistics \& Transportation Review, 69(3), 2014, pp.160-179.

18. Wang, H. J., Wang, J., Ma, S. H., "Decision-making for emergency materials dynamic dispatching based on fuzzy demand and supply". Chinese Journal of Management Science, 22(1), 2014, pp.55-64.

19. Yi, H. N., "Research on optimization of emergency rescue material dispatching". Journal of Gansu Sciences, 26(2), 2014, pp.110-113.

20. Chen, G. T., Shuai, B., "Emergency supply scheduling optimization under demand uncertainty". Journal of Transportation Engineering and Information, 2(3), 2015, pp.22-26.

21. Zhang, X. S., Wang, W., Jiang, H. S., "Study on optimum emergency material dispatching based on network reliability". Logistics Technology, 31(9), 2012, pp.235-237.

22. Li, X. X., Hao, Y. W., Zhao, Z., "Multi-objective emergency material dispatch model based on maximum flow". China Journal of Emergency Resuscitation and Disaster Medicine, 11(10), 2016, pp.964-967.

23. Tang, W. Q., Zhou, L., Guo, Q. Y., "Grey multi-objective programming model for emergency material dispatching in initial stage of emergency". China Safety Science Journal, 26(4), 2016, pp.155-160.

24. He, S. S., Zhu, W. H., Zhuang, X. Q., "Research on multi-stage distribution routes of emergency supply based on post-disaster road rehabilitation". Computer Simulation, 33(3), 2016, pp.167-171.

25. Shen, Z. P., Gao, J., "GO methodology and improved quantification of system reliability". Journal of Tsinghua University (Science and Technology), 39(6), 1999, pp.15-19.

26. Du, L. J., "Multi-objective optimization of relief distribution in emergency". Doctor thesis of Huazhong University of Science and Technology, China, 2015, pp.25-115. 